\title{
SULLA RISULTANTE DI DUE POLINOMI IN UNA VARIABILE.
}

\author{
Nota di Francesco Gecioni (Livorno).
}

Adunanza del 25 maggio 1913 .

Scopo di questa breve Nota è di dare una dimostrazione diretta della identità fra la risultante di due funzioni razionali intere in una variabile data dal determinante di Sylvester e la risultante medesima calcolata col metodo offerto dalla teoria delle funzioni simmetriche delle radici di una equazione algebrica. Una dimostrazione diretta di tale identità è data dal Netro ${ }^{1}$ ), ed un'altra dal KöNig ${ }^{2}$ ). In queste dimostrazioni vengono però fatte alcune particolari ipotesi sulle due funzioni, ipotesi che poi vengono eliminate in virtù del principio di identità. Nella dimostrazione che segue non viene invece fatta alcuna ipotesi speciale, e vengono adoperati mezzi di dimostrazione elementarissimi.

Siano

$$
\begin{aligned}
& f(x)=a_{0} x^{n}+a_{1} x^{n-1}+\cdots+a_{n-1} x+a_{n}, \\
& g(x)=b_{0} x^{m}+b_{1} x^{m-1}+\cdots+b_{m-1} x+b_{m}
\end{aligned}
$$

due funzioni razionali intere nella variabile $x$, e siano $x_{\mathrm{t}}, x_{2}, \ldots, x_{n}$ le $n$ radici, uguali o distinte, della equazione

$$
f(x)=o \text {; }
$$

indicando con $R_{f, g}$ il seguente determinante (di Sylvester) di ordine $n+m$

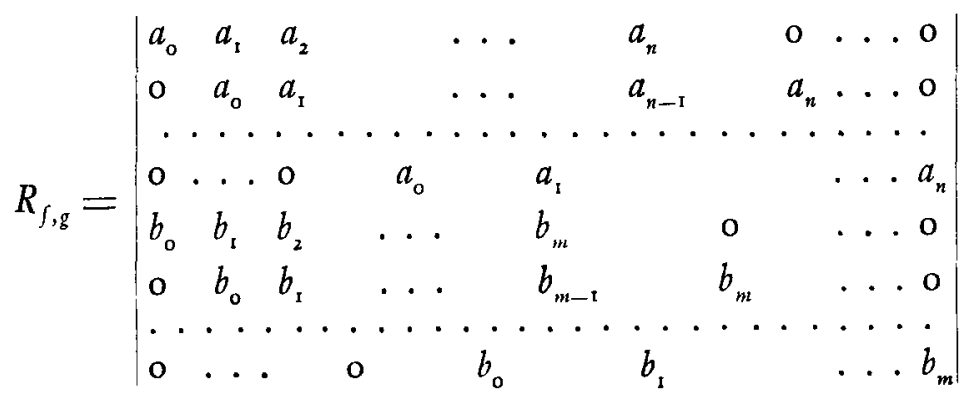

I) E. NeTTO, Vorlesungen über Algebra (Leipzig, Teubner), Bd. I (1896), p. I5 I.

2) J. KठNIG, Einleitung in die allgemeine Theorie der algebraischen Grössen (Leipzig, Teubner, 1903), p. 122. 
l'identità che si tratta di dimostrare è la seguente:

$$
R_{f, g}=a_{\mathrm{o}}^{m} g\left(x_{1}\right) g\left(x_{2}\right) \ldots g\left(x_{n}\right) .
$$

Procediamo per questo per induzione rispetto al grado $n$ della funzione $f(x)$, e verifichiamo quindi intanto che la identità (2) è vera per $n=\mathrm{I}$. In tal caso il determinante $R_{f, g}$ assume la forma

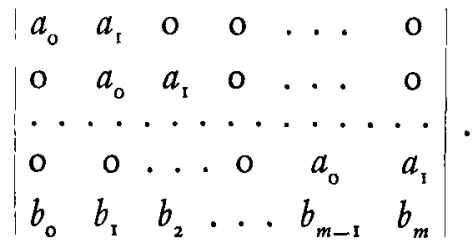

Aggiungiamo in questo determinante alla seconda colonna la prima moltiplicata per $x_{1}=-\frac{a_{1}}{a_{0}}$; indi, nel determinante cost modificato, aggiungiamo alla terza colonna la seconda moltiplicata pure per $x_{1}$, e cosi via di seguito; il determinante superiore si trasforma allora nel seguente

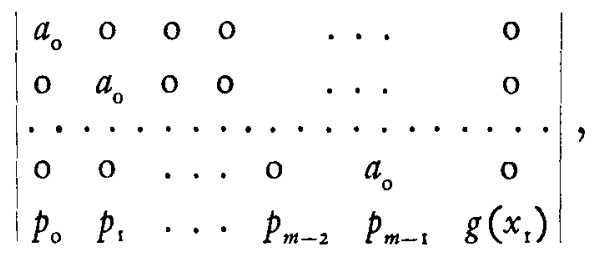

dove $p_{0}, p_{1}, \ldots, p_{m-1}$ rappresentano $\mathrm{i}$ coefficienti del quoziente $\frac{g(x)}{x-x_{1}}$ :

$$
p_{i}=b_{0} x_{1}^{i}+b_{1} x_{1}^{i-1}+\cdots+b_{i-1} x_{\mathrm{r}}+b_{i} \quad(i=0,1,2, \ldots, m-1) .
$$

$\mathrm{Ma}$ tale determinante è uguale ad $a_{0}^{m} g\left(x_{\mathrm{t}}\right)$, onde in questo caso, cioè per $n=\mathrm{I}$, la identità (2) è dimostrata.

Supposto allora che la identità da dimostrare in generale sia vera per una funzione $f(x)$ di grado $n-\mathrm{I}$, dimostriamo che è vera pure quando il grado della funzione $f(x)$ è $n$.

Consideriamo perciò il determinante $R_{f, g}$ dato dalla (I), ed in esso aggiungiamo alla seconda colonna la prima moltiplicata per $x_{1}$; indi, nel determinante cosi trasformato, aggiungiamo alla terza colonna la seconda moltiplicata pure per $x_{1}$; e cosi seguitiamo fino all'ultima colonna. Il determinante $R_{f, g}$ si trasforma cosi nel seguente

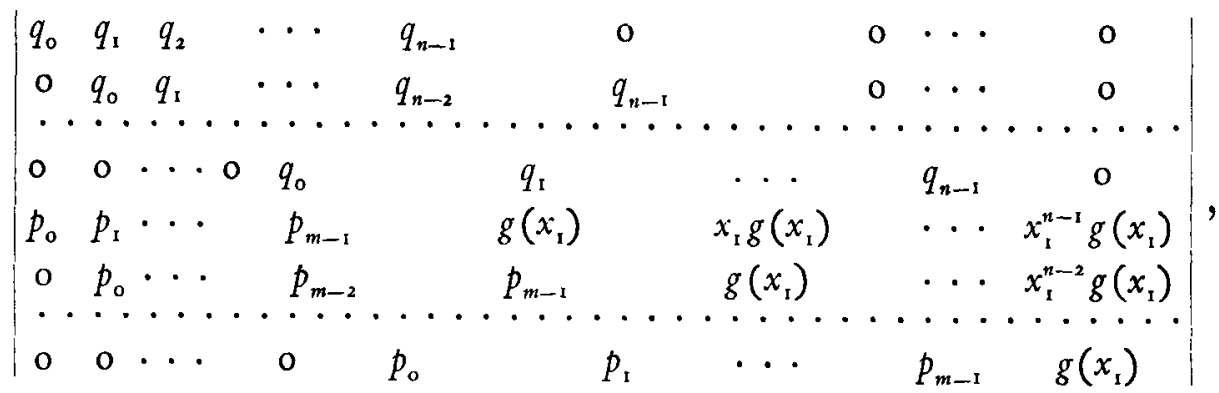


dove $q_{0}, q_{1}, \ldots, q_{n-1}$ indicano $\mathrm{i}$ coefficienti del quoziente $q(x)=\frac{f(x)}{x-x_{1}}$, e $p_{0}$, $p_{\mathrm{r}}, \ldots, p_{m-1}$ i coefficienti del quoziente $\frac{g(x)}{x-x_{\mathrm{r}}}$ :

$$
\begin{aligned}
& q_{i}=a_{0} x_{1}^{i}+a_{1} x_{1}^{i-1}+\cdots+a_{i-1} x_{1}+a_{i} \quad(i=0,1,2, \ldots, n-1) \\
& p_{b}=b_{0} x_{1}^{h}+b_{1} x_{1}^{b-1}+\cdots+b_{h-1} x_{1}+b_{h} \quad(b=0,1,2, \ldots, m-1) .
\end{aligned}
$$

In questo determinante sottragghiamo dalla $(m+1)^{\text {esima }}$ riga la $(m+2)^{\text {esima }}$ moltiplicata per $x_{1}$; dalla $(m+2)^{\text {esima }}$ la $(m+3)^{\text {esima }}$ moltiplicata pure per $x_{1}$, e cosi via; otteniamo cosi :

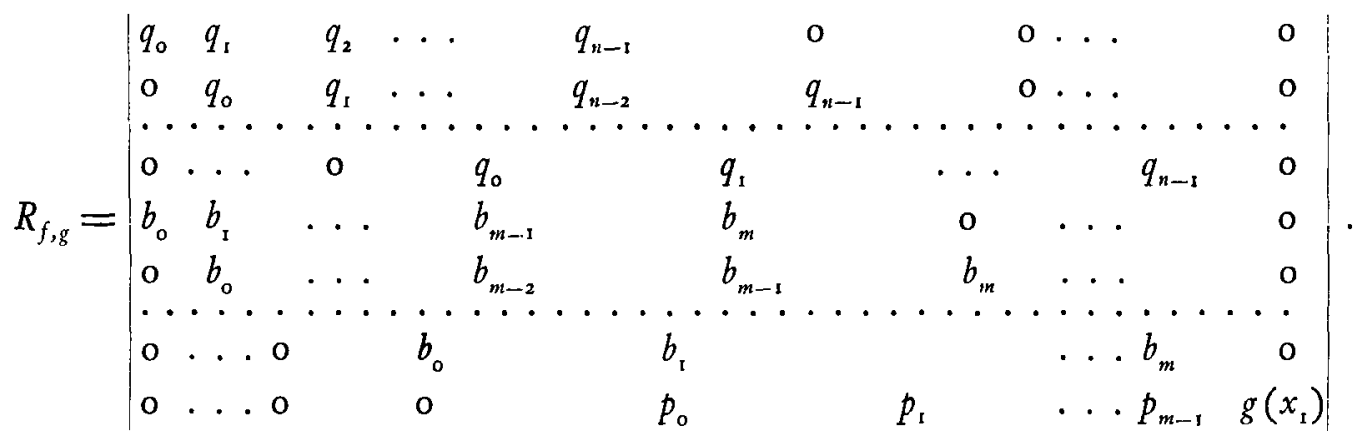

Sviluppando ora questo determinante per gli elementi dell'ultima colonna abbiamo

$$
R_{f, g}=g\left(x_{1}\right) R_{q, g}
$$

dove $R_{q, g}$ rappresenta la risultante, scritta col determinante di Sylvester, dei polinomi $q(x)=\frac{f(x)}{x-x_{\mathrm{t}}}$ e $g(x)$. Ma $q(x)$ ha il grado $n$ - I rispetto ad $x$; quindi per il determinante $R_{q, y}$ la identita in questione è per ipotesi verificata, e si ha perciò (essendo $\left.q_{\mathrm{o}}=a_{\mathrm{o}}\right)$

risulta dunque, sostituendo,

$$
R_{q, g}=a_{\mathrm{o}}^{m} g\left(x_{2}\right) g\left(x_{3}\right) \ldots g\left(x_{n}\right)
$$

$$
R_{f, g}=a_{\mathrm{o}}^{m} g\left(x_{1}\right) g\left(x_{2}\right) \ldots g\left(x_{n}\right),
$$

A complemento della dimostrazione esposta è facilissimo dedurre anche l'identità

$$
R_{f, g}=(-\mathrm{I})^{m n} b_{\mathrm{o}}^{n} f\left(y_{\mathrm{r}}\right) f\left(y_{2}\right) \ldots f\left(y_{m}\right)
$$

dove $y_{1}, y_{2}, \ldots, y_{m}$ sono le radici dell'equazione $g(x)=0$. Scambiando infatti fra loro le righe del determinante $R_{f, g}$ dato dalla (I) in modo che vengano a scambiarsi la matrice delle prime $m$ righe e quella delle altre $n$, si ottiene evidentemente il determinante $R_{g, f}$, e risulta quindi

$$
R_{f, g}=(-\mathrm{I})^{m n} R_{g, f}
$$

$\mathrm{Ma}$ per la formula ora dimostrata si ha

è dunque

$$
R_{g, f}=b_{\mathrm{o}}^{n} f\left(y_{\mathrm{t}}\right) f\left(y_{2}\right) \ldots f\left(y_{m}\right) ;
$$

come volevamo ottenere.

$$
R_{f, g}=(-\mathrm{I})^{m n} b_{\mathrm{o}}^{n} f\left(y_{\mathrm{t}}\right) f\left(y_{2}\right) \ldots f\left(y_{m}\right)
$$

Livorno, is maggio I9I3.

Francesco CECIONI. 